\title{
Fabricating Millifluidic Reaction-Diffusion Devices: Droplet-in-Oil Networks Structured by Laser Cutting
}

\author{
Kai Ming Chang, Maurits R.R. de Planque and Klaus-Peter Zauner \\ Electronics and Computer Science \\ University of Southampton \\ Southampton, SO17 1BJ, United Kingdom
}

\begin{abstract}
All known forms of life utilise information processing to maintain their complex organisation. In contrast to conventional information technology built on solid-state semiconductor devices, biological information processing is built on transformations through chemical reactions and interactions mediated by diffusion. The theoretical understanding of reaction-diffusion computing as well as prototype implementations have progressed in parallel over the past decades. We report here on a technique for studying spatially structured networks in which chemicals are compartmentalised as droplets-in-oil and laser-cut topologies impose spatial structure. Experiments with halogen displacement reactions demonstrate that the feature size achievable with laser cutting is well suited to practical diffusion time scales. Further advantages of the technique are the optical accessibility, enabling readout from bromine and iodine production, diffusion and indicator reactions, and the good chemical compatibility between the compartmentalisation medium (oil) and the structuring medium (PMMA), while the fast turn-around times enable rapid topology optimisation.
\end{abstract}

Keywords-compartmentalisation; microfluidics; lab-onchip; reaction networks; molecular computing; coupled microreactors; signal cascade

\section{INTRODUCTION}

The study of reaction-diffusion systems from an information processing perspective goes back to the dawn of computer science. While Turing considered how the interplay of compound transformation and compound diffusion can impose structure on space [1,2], in many subsequent studies spatial structure was imposed on the system. For example, in Kuhnert's seminal work, spatially structured illumination levels were used to provide input information to a chemical image processing system that was based on a light-sensitive variant of the BelousovZhabotinsky (BZ) reaction [3]. More commonly, such chemical oscillator systems are guided towards the desired functionality by imposing a two-dimensional structure through patterned illumination [4]. In these studies, local concentration gradients are generated by photo-responsive molecules, giving rise to spatially structured diffusion.

Another approach to structure reaction-diffusion systems is the placement of physical barriers. The active medium can fill channel-connected wells as a continuous phase $[5,6]$, or compartmentalisation can be achieved by self-organisation within the physical structure [7]. An example of the latter scenario is a chain of droplets separated by oil plugs inside a glass capillary of $150 \mu \mathrm{m}$ diameter [8]. In such a two-phase system the oil serves as a semipermeable barrier between the aqueous compartments. In this one-dimensional chain, synchronised oscillations were observed depending on the droplet spacing, which determines the strength of the diffusive coupling between the aqueous compartments. The spatial arrangement of these droplets is constrained by the capillary, with the chemically inert glass inner surface imposing a linear network structure.

Two-dimensional droplet-in-oil networks can be achieved with microfabrication or micromachining methods [9]. Microfabrication with UV-Vis lithography enables very small feature sizes, down to $\sim 10 \mu \mathrm{m}$, in a layer of photoresist, from which a microfluidic device can be created by replica moulding [10]. However this approach requires quite extensive laboratory (if not clean room) infrastructure, each design iteration necessitates a new photomask, and the standard replica moulding material poly(dimethyl siloxane) (PDMS) swells in oil while it can also absorb ions that participate in the chemical reactions of interest $[11,12]$. On the other hand, micromachining approaches such as 3Dprinting and laser cutting only require a single piece of equipment, while features down to $\sim 200 \mu \mathrm{m}$ can be realised in a range of materials. These cost-effective methods are also flexible because a new design only requires a new software input, which is favourable for the rapid prototyping of reaction-diffusion devices. With features in the millimetre range such devices trade-off a reduction of the amount of chemicals for easier device fabrication. Because of their feature size, we refer to such devices as millifluidic rather than microfluidic.

We have previously described $\mathrm{BZ}$ wave propagation in 3D-printed reaction vessels where the channels and the wells contained BZ medium in direct contact with the air $[5,13]$. The reaction-diffusion medium was physically structured but a two-phase system was not implemented. In the present paper, we describe millifluidic devices fabricated by laser cutting of the optically transparent oil-compatible polymer poly(methyl methacrylate) (PMMA). The wells in these devices define the position of various aqueous reaction media, while the channels that connect the wells contain hexadecane oil as the diffusion medium. We use halogen displacement reactions [14], where molecular bromine or iodine are generated in specific droplets and diffuse through the oil to react in other wells. These reactions are visualised 
as colour changes, enabling rapid optimisation of the reaction-diffusion system both in terms of the chemical composition and in terms of the network topology as defined by laser-cut PMMA prototype devices.

\section{MATERIALS AND METHODS}

\section{A. Bromine and iodine formation}

Bromine $\left(\mathrm{Br}_{2}\right)$ and iodine $\left(\mathrm{I}_{2}\right)$ were produced in aqueous droplets under a layer of hexadecane oil (99\%, SigmaAldrich, Dorset, UK). Molecular bromine is soluble in water and in hexadecane, giving a yellow-orange or brown colour. Molecular iodine is soluble in hexadecane, giving a purple colour, but is virtually insoluble in aqueous solution. However, in an aqueous solution of potassium iodide (KI), iodine converts to the water-soluble triiodide ion $\left(\mathrm{I}_{3}^{-}\right)$, which appears yellow at lower and brown at higher concentration.

Bromine-generating droplets are essentially composed of a non-oscillating BZ mixture that lacks the substrate species (e.g. malonic acid). As shown in Table $\mathrm{I}$, the reaction components were split into two half-mixtures to prevent the reaction described in (1) to start prematurely, i.e. prior to oil immersion. Malonic acid can be added to stop this reaction. This initiates Belousov-Zhabotinsky oscillations, which consume most of the bromine before it can diffuse into the surrounding oil [8,15]. All these chemicals were from Sigma-Aldrich.

$$
\begin{aligned}
& 5 \mathrm{KBr}(\mathrm{aq})+3 \mathrm{H}_{2} \mathrm{SO}_{4}(\mathrm{aq})+\mathrm{NaBrO}_{3}(\mathrm{aq}) \rightarrow \\
& 3 \mathrm{Br}_{2}(\mathrm{~g})+3 \mathrm{~K}_{2} \mathrm{SO}_{4} / \mathrm{Na}_{2} \mathrm{SO}_{4}(\mathrm{aq})+3 \mathrm{H}_{2} \mathrm{O}(\mathrm{l})
\end{aligned}
$$

To produce molecular iodine, the same procedure was followed, except that $\mathrm{KBr}$ was replaced with $\mathrm{KI}$.

\begin{tabular}{|c|c|c|c|}
\hline $\begin{array}{l}\text { Half- } \\
\text { mixture }\end{array}$ & Chemical Stock Solution & $\begin{array}{l}\text { Volume } \\
(\mu \mathrm{L})\end{array}$ & $\begin{array}{c}\text { Concentration } \\
(\mathbf{m M})^{\mathrm{a}}\end{array}$ \\
\hline A & $\begin{array}{l}2.5 \mathrm{M} \text { sulphuric acid } \\
\left(\mathrm{H}_{2} \mathrm{SO}_{4}\right)\end{array}$ & 120 & 692 \\
\hline A & Deionised water $\left(\mathrm{H}_{2} \mathrm{O}\right)$ & 313.3 & - \\
\hline B & $\begin{array}{l}1.5 \mathrm{M}_{\text {sodium bromate }} \\
\left(\mathrm{NaBrO}_{3}\right)\end{array}$ & 66.7 & 176 \\
\hline B & $\begin{array}{l}1.0 \mathrm{M} \text { potassium bromide } \\
(\mathrm{KBr})\end{array}$ & 500 & 882 \\
\hline
\end{tabular}

TABLE I. REACTION MIXTURE FOR BROMINE GENERATION

${ }^{\text {a. }}$ Concentration in the complete reaction mixture, consisting of equal volumes of the half-mixtures

\section{B. Indicator droplets for bromine and iodine diffusion}

Droplets of aqueous KI solution were used to indicate bromine diffusion through the hexadecane phase. When molecular bromine arrives at the KI droplet it participates in the halogen displacement reaction [14] shown in (2). As mentioned above and as shown in (3), iodine subsequently reacts to $\mathrm{I}_{3}^{-}$because of the presence of $\mathrm{KI}$, changing the initially colourless KI droplet to yellow. Additionally, when a large amount of iodine is generated in the KI droplet, the iodine can diffuse into the surrounding hexadecane, colouring the oil purple.

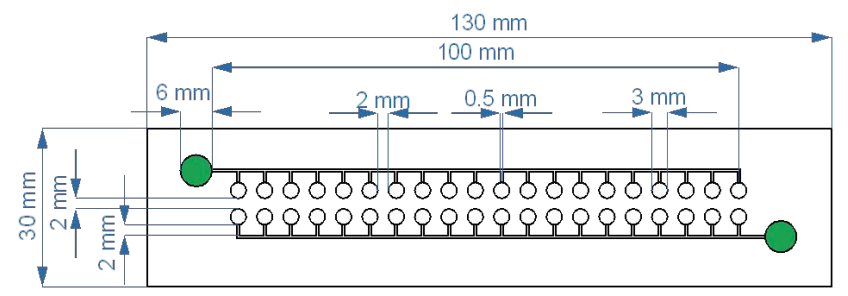

Fig. 1. In this example of a laser cutter design file, the source chamber for iodine generation and the side wells for the starch-iodine reaction are defined as circular outlines. The two main channels and their side channels, to be filled with hexadecane as diffusion medium, are defined as rectangular outlines. The laser cutter only removes PMMA along the black lines, after which free-standing material is pushed out of the PMMA sheet. Filled circles (green) show iodine supplying wells while open circles show starch reaction wells.

$$
\begin{aligned}
& 2 \mathrm{KI}(\mathrm{aq})+\mathrm{Br}_{2}(\mathrm{~g}) \rightarrow 2 \mathrm{KBr}(\mathrm{aq})+\mathrm{I}_{2}(\mathrm{aq}) \\
& \mathrm{KI}(\mathrm{aq})+\mathrm{I}_{2}(\mathrm{aq}) \leftrightarrow \mathrm{KI}_{3}(\mathrm{aq})
\end{aligned}
$$

To indicate iodine diffusion through the oil, aqueous droplets of solubilised starch can be used. When KI is also present, the generated $\mathrm{I}_{3}{ }^{-}$ions insert into amylose helices, and these triiodide-amylose complexes cause an intense purpleblack colour. In the absence of KI this darkening of the starch solution is significantly slower but does eventually occur. Starch droplets do not change from colourless to black upon exposure to bromine. Instead, bromine diffusion colours these droplets yellow-orange. This colour change is not permanent because the bromine will continue to diffuse through the oil phase. A starch stock solution was obtained by mixing $250 \mathrm{mg}$ of corn flour (Brown and Polson, UK) with $1 \mathrm{~mL}$ of warm deionised water. This was then diluted to $10 \mathrm{mg} / \mathrm{mL}$ starch using pure water or an aqueous KI solution, resulting in a somewhat opaque dispersion, reflecting the limited water solubility of starch.

\section{PMMA device fabrication}

An Epilog Mini 30W CO 2 laser cutter (Epilog Laser, CO, USA) was used to cut channels and wells out of PMMA sheets (TechSoft Ltd., Bodelwyddan, UK), with the power and speed of the 5000 pulses per inch laser optimised to create a hairline cut all the way through the sheet. For example, after laser cutting the outline of a circle, a well is created by pushing the PMMA material inside the circle out of the sheet. The devices were designed by defining the outlines of the channels and wells in CorelDRAW Graphics Suite (Corel Corporation, Ottawa, CA) software. A sample of such a design is shown in Fig. 1. Each device is composed of a $5 \mathrm{~mm}$ thick PMMA sheet with the channels cut all the way through and a $1.5 \mathrm{~mm}$ thick solvent-bonded [16] PMMA baseplate which constitutes a liquid-tight channel floor. The channel layouts are $0.5 \mathrm{~mm}$ in width, which results in an actual channel width of $0.7-0.9 \mathrm{~mm}$ due to the kerf of the laser-cutter. Narrower channels are avoided because these can block during solvent bonding. The dimensions given for the wells in the following sections always refer to the dimension in the layout file and the actual opening that is cut is $0.2 \mathrm{~mm}$ wider along each edge, i.e. a $3 \mathrm{~mm}$ well has an 
actual diameter of $3.4 \mathrm{~mm}$. Compared to the laser ablation of the channels into a single block of PMMA, this method results in a uniform channel depth and a smooth and transparent channel floor. Moreover, because the volume of material the laser has to burn away is restricted to the cut, i.e. the laser has to trace only the outline of the channels and wells once rather than repeatedly scan in a fine raster over the area to be etched away, it is typically two orders of magnitude faster.

For solvent bonding, dichloromethane (Bondrite Adhesives Ltd., Leicestershire, UK) was pipetted into the channels and allowed to spread between the channel-defining top PMMA sheet and the PMMA baseplate by capillary action. The baseplate was cut $5 \mathrm{~mm}$ wider than the top sheet, with thinly etched lines on the periphery of the bonding area, to ensure that the solvent remained in contact with the outer edges of the device. Hand pressure was applied and extra solvent was added to ensure a liquid-tight bond. Excess solvent was subsequently allowed to evaporate in a fume hood. Finally, the devices were exposed to trichloro( $1 H, 1 H, 2 H, 2 H$-perfluorooctyl)silane (97\%, SigmaAldrich) vapour in a desiccator to make the PMMA surface more hydrophobic, which facilitates wetting and hence channel filling by the hexadecane oil. The solvent gives rise to irregular bubble patterns in the glued areas, which can be observed in Figs. 3, 5 and 7. These are outside the channels and wells however, and do not affect the experimental observations.

\section{Imaging}

The PMMA devices were placed on top of a uniform LED backlight (MiniSun A4 LED Modern Ultra-Slim Art Craft Design LightPad, MiniSun UK) that was covered by a borosilicate glass worktop saver. After filling the device with hexadecane and pipetting the appropriate solutions in the different wells, the diffusion of bromine and iodine through the oil channels and the reactions inside the wells were captured by a Prosilica GX2300C CCD camera with a $2336 \times 1752$ pixel sensor (Allied Vision, Germany). Allied Vision's Vimba software was used to operate the camera, saving images as BMP files at a frame rate of up to $0.4 \mathrm{~Hz}$. The images were processed with ImageJ software (National Institutes of Health, USA) to generate space-time plots by cropping a row of wells of each frame in a recording and arranging these cropped images next to each other, in the order with which the images were taken.

\section{RESULTS AND DISCUSSION}

\section{A. Observation of bromine diffusion and KI reaction}

The generation and diffusion of bromine was first tested in a glass Petri dish. A strip of fiberglass filter paper was positioned in the middle of a $90 \mathrm{~mm}$ diameter dish filled with $5 \mathrm{~mL}$ of hexadecane. This fiberglass strip was saturated with $140 \mu \mathrm{L}$ of bromine-generating solution (Table I), which protruded from the paper reservoir as two distinct droplets. These hexadecane-submerged droplets turned yellow-orange, confirming that bromine was produced. As shown in Fig. 2, the hexadecane surrounding the droplets and the strip also turned yellow-orange, indicating bromine diffusion through the oil. After $\sim 1$ minute and 10 minutes the hexadecane started to colour at $\sim 6 \mathrm{~mm}$ and $15 \mathrm{~mm}$ distance, respectively, from the strip or droplet edges, implying a decrease of the bromine diffusion speed over time. After $\sim 20$ minutes the colour intensity in the oil and in the aqueous source droplets started to diminish and eventually both oil and droplets returned to their initial colourless state.

Several $10 \mu \mathrm{L}$ droplets of $1 \mathrm{M} \mathrm{KBr}$ solution and droplets of $1 \mathrm{M}$ KI solution were placed at $\sim 1 \mathrm{~cm}$ distance from the strip. These droplets were initially colourless. Fig. 2 shows that the $\mathrm{KBr}$ droplets remained uncoloured, implying that the surrounding oil did not deliver an appreciable amount of bromine to these droplets. However, the KI droplets did turn yellow, confirming the formation of $\mathrm{I}_{3}{ }^{-}$according to (3) following the halogen displacement reaction (2). Over time, KI droplets closer to the bromine-producing source droplets appeared darker in colour, indicating uptake of a larger amount of hexadecane-diffused bromine than KI droplets positioned at a larger distance. Close inspection of these darker droplets revealed a faint purple halo, indicating diffusion of excess iodine into the hexadecane. The KI droplets did not discolour over the duration of the experiment, most likely because the $\mathrm{I}_{3}{ }^{-}$ion is not soluble in apolar media such as hexadecane.

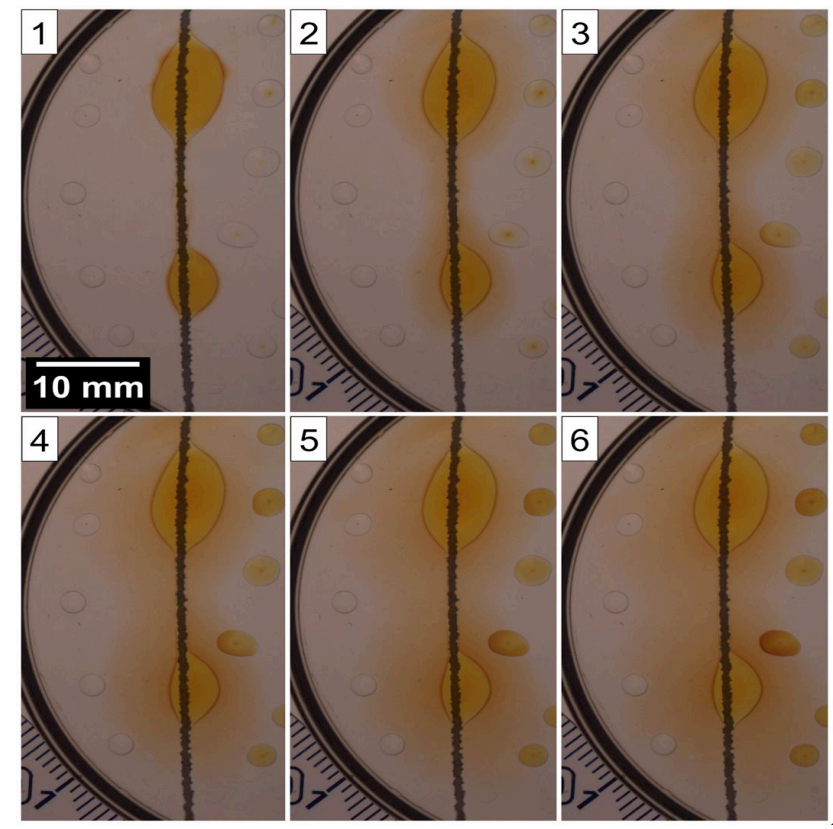

Fig. 2. Visualisation of bromine diffusion through bulk hexadecane. A paper strip saturated with the bromine-generating reaction mixture, which protrudes as two droplets from the strip, is placed in a Petri dish and covered by hexadecane. Bromine diffusion through the oil is visible as an expanding yellow-orange zone. Several $10 \mu \mathrm{L} \mathrm{KBr}$ droplets are positioned on the left side of the strip and several KI droplets on the right side. Both types of droplets adsorb onto the glass surface, and stay in position. The colour development in the KI droplets indicates formation of the triiodide ion. The images are taken at 10 second intervals. 


\section{B. Iodine diffusion timer device}

The reaction of iodine with KI-supplemented starch solution was demonstrated in the millifluidic PMMA device depicted in Fig. 1. This consists of a source chamber of 6 $\mathrm{mm}$ diameter from which a $10 \mathrm{~cm}$ long channel protrudes. Along this main channel 20 side channels of $2 \mathrm{~mm}$ length are positioned at regular intervals, each connecting to a well of 3 $\mathrm{mm}$ diameter. The intention of this design is to establish over which main-channel distance iodine generated in the source chamber can trigger a starch reaction in a side well. A second well-channel network, in the reverse orientation, is included in the same PMMA device.

First, each of the two reaction-diffusion networks of this device was filled with $1.5 \mathrm{~mL}$ hexadecane. Then $20 \mu \mathrm{L}$ of a $1 \%(\mathrm{w} / \mathrm{v})$ starch solution in $0.5 \mathrm{M} \mathrm{KI}$ was pipetted in each side well, followed by $40 \mu \mathrm{L}$ of an iodine-generating reaction mixture (see Table I but with KI rather than $\mathrm{KBr}$ ) in the source chamber. Immediately upon mixing the two halfsolutions together in the source chamber, the reaction mixture droplet turned yellow-black and a purple front started to move through the hexadecane-filled channel, confirming the formation and the oil diffusion of iodine.
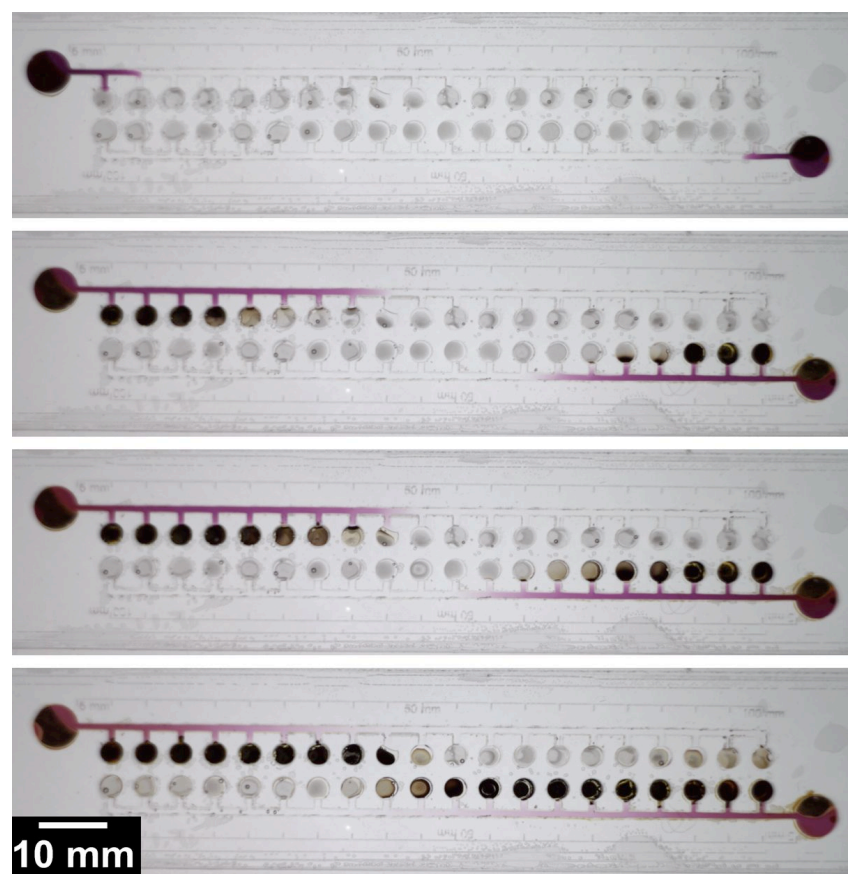

Fig. 3. Diffusion of iodine through a hexadecane channel towards an array of starch-KI wells, for two independent reaction networks. The first image (recorded immediately, i.e. $0 \mathrm{~min}$, after mixing the iodine-generating mixture into the source chambers) shows the iodine molecules, clearly visible as an intense purple colouring of the hexadecane, diffusing out of the source chamber. In the second image $(30 \mathrm{~min})$ iodine is visible in the main channel up to the 9th side well, while the first 5 side wells have considerably darkened because of the starch reaction. The third image $(60$ min) indicates that iodine diffusion through the main channel has slowed down while the starch reaction in the iodine-exposed side wells continues. The final image (300 min) shows the furthest effective diffusion distance; note that the purple iodine colour of the oil fades over time.
Interestingly, when observed through the side wall of the device, the iodine initially only diffuses along the bottom of the main channel, probably reflecting liquid iodine's 4-fold higher density than hexadecane.

Fig. 3 shows that iodine diffusing through the hexadecane in the main channel enters the side channels and causes the side wells to turn black. This colour change happened immediately upon the arrival of iodine at the side wells, suggesting rapid triiodide formation and amylose interaction. There is a short delay for the colour to diffuse through the droplet, however, and this contributes to well 5 appearing to turn black sooner than well 4, which is closer to the iodine source in the space-time plot (Fig. 4). The iodide$\mathrm{KI}$-amylose reaction in the side wells followed the diffusion of iodide through the main channel, presenting a convenient read-out of the iodine diffusion speed and of the maximum diffusion distance from the source chamber. The space-time plot in Fig. 4 shows that the iodine diffusion speed decreases at a larger distance from the iodine source and that the maximum diffusion distance is $60 \mathrm{~mm}$ for the lower channel and $50 \mathrm{~mm}$ for the upper channel. The diffusion speed, based on the onset of side well colouring with this particular device geometry and amount of iodine-generating reaction mixture, is estimated at 0.9 and $1 \mathrm{~mm} / \mathrm{min}$ at $15 \mathrm{~min}, 0.7$ and 0.6 $\mathrm{mm} / \mathrm{min}$ at $30 \mathrm{~min}, 0.5$ and $0.3 \mathrm{~mm} / \mathrm{min}$ at $45 \mathrm{~min}$, then 0.4 and $0.2 \mathrm{~mm} / \mathrm{min}$ at $60 \mathrm{~min}$, and 0.2 and $<0.1 \mathrm{~mm} / \mathrm{min}$ at 90 $\mathrm{min}$, for the lower and upper channels respectively.

The devices used in the experiments described in this section and in the following section comprise two identical reaction-diffusion networks and thus illustrate the variability that is to be expected in practical implementations of such devices. The experiments described in this paper are typical samples from tests with variations of the well spacing, well size and well number; the results across these variations were consistent.

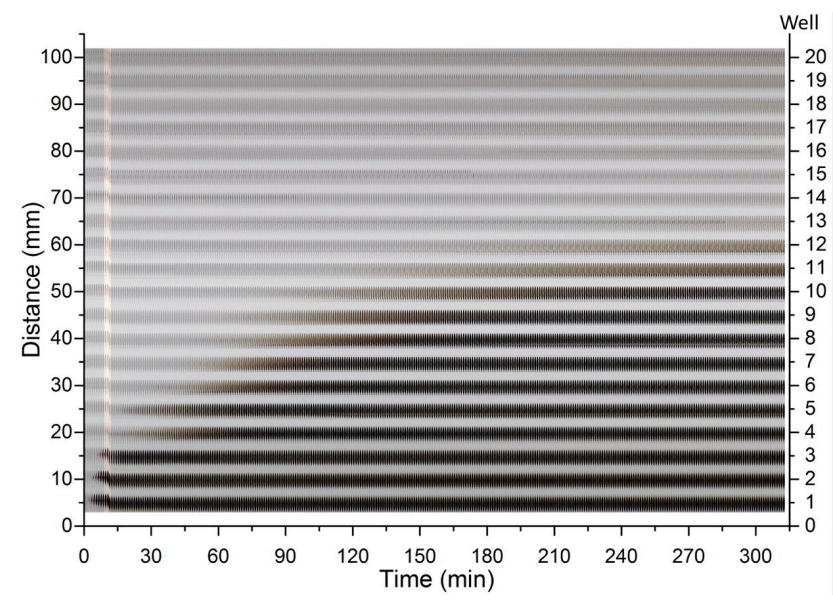

Fig. 4. Space-time plot showing the starch colour development in the 20 side wells of the lower network shown in Fig. 3. Centre-of-well slices were taken every minute. The diffusion of iodine through the main channel slows down after $\sim 2$ hours. After $\sim 5$ hours starch colouring is not triggered in chambers 13-20; the maximum diffusion distance of iodine is $60 \mathrm{~mm}$. 


\section{Interleaved side wells}

The initial diffusion characterisation device was then adapted to a reduced main channel length of $50 \mathrm{~mm}$, ensuring that iodine diffusing through the hexadecane could, eventually, reach all side wells of the device. As an additional modification, we implemented only a single row of side wells, which are alternately connected to the top or to the bottom main channel (see Fig. 5). The dimensions of the wells and side channels are kept the same as in Fig. 1. Each side of this device was filled with $250 \mu \mathrm{L}$ of hexadecane, after which $20 \mu \mathrm{L}$ of the starch-KI solution was pipetted into each side well and then $40 \mu \mathrm{L}$ of the iodine-generating reaction mixture was pipetted into each source chamber.

As in the previous experiment, iodine diffusion from the source chamber into the main channel and in the side channels was visible as a purple colouration of the hexadecane oil, and iodine delivery to the side wells resulted in a purple-black colour development due to the KI-mediated reaction with amylose. The space-time plot of the single row of side wells in this device is shown in Fig. 6. Because the odd-numbered and the even-numbered wells are connected to different main channels, with an opposite location of the iodine source chamber, this plot represents a more complex reaction-diffusion output than the space-time plot (Fig. 4) of the initial device. As observed earlier, the iodine diffusion speed is considerably reduced after $\sim 100$ minutes, at which point the iodine has diffused $\sim 40 \mathrm{~mm}$ into the main channel.

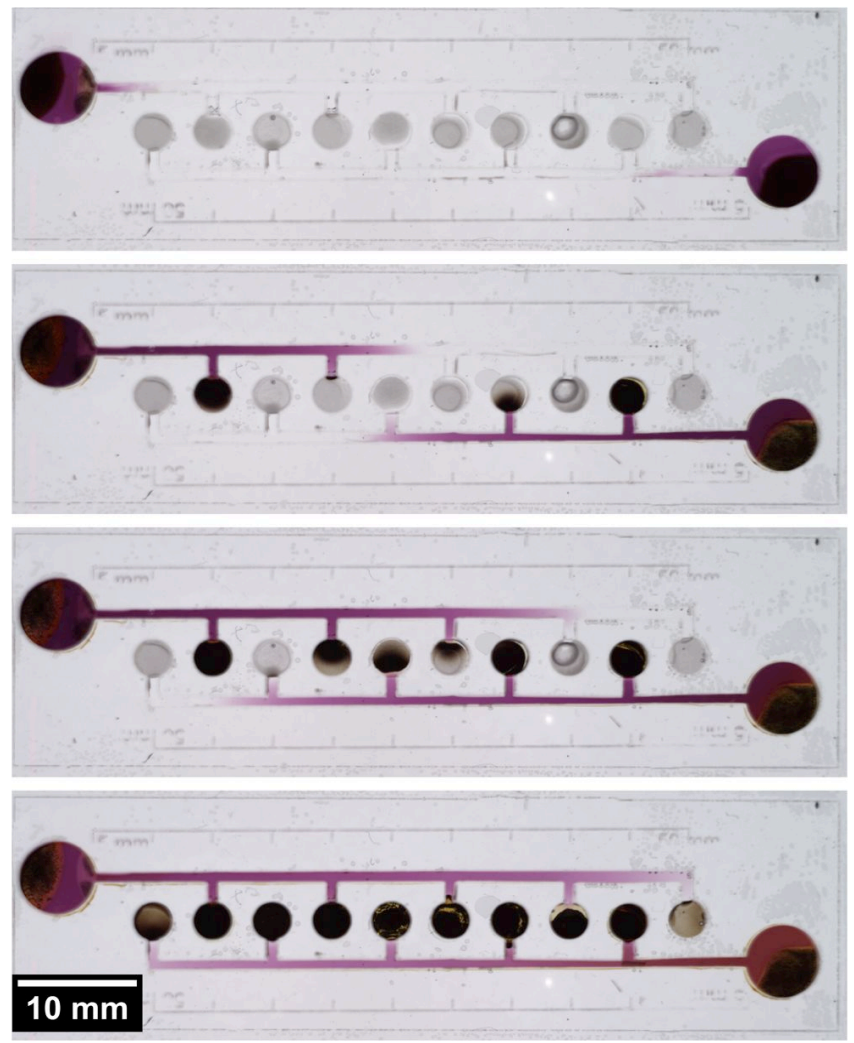

Fig. 5. Starch-iodine reactions in an array of side wells that are alternately in contact with an upper or a lower main channel, each with its own iodinegenerating source chamber. The images (top to bottom) were taken at $\sim 10$, 30, 50 and 190 minutes after the onset of iodine generation.

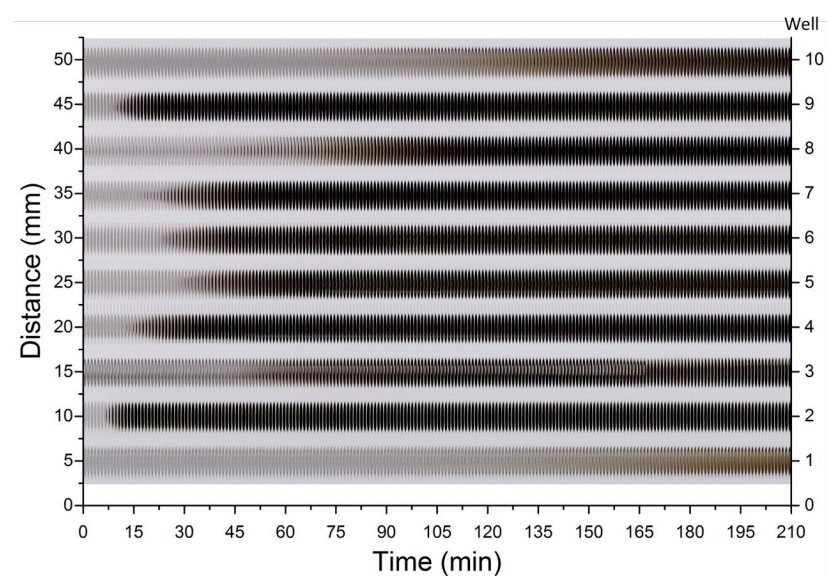

Fig. 6. Space-time plot showing the starch colour development in the 10 side wells of the device shown in Fig. 5. Centre-of-well slices were taken every minute. The space-time plot does not present a linear reaction progression because of the 'interleaved' topology of the network, with wells $1,3,8$ and 10 delayed from turning black.

\section{Three-component reaction-diffusion network}

As a final demonstration of PMMA millifluidics, we sought to implement a reaction network of three different chemical reaction nodes that are coupled by hexadecane diffusion of both bromine and iodine. In the tree-shaped design shown in Fig. 7, the lower (referring to the position in the images) chamber contains the bromine-generating reaction mixture (Table I), the middle chamber is filled with KI solution, and each of the five top wells contains starch solution. Bromine diffusion from the bottom chamber to the middle chamber should give rise to iodine formation through the halogen displacement reaction shown in (2). The iodine molecules generated in the KI chamber should then diffuse through the hexadecane channels to the top wells, colouring the starch solution black.

This sequential reaction scheme requires a sufficient amount of bromine to reach the KI chamber, otherwise the halogen displacement reaction in this droplet cannot generate enough iodine for a downstream starch reaction. Given the limited efficiency of bromine diffusion observed in the bulk oil experiment (Fig. 2), we performed several design iterations, leading to the implementation of a relatively large well (4 $\mathrm{mm}$ diameter) for the bromine-generating reaction mixture, connected with a relatively short channel of $2.5 \mathrm{~mm}$ length to the $\mathrm{KI}$ chamber ( $3 \mathrm{~mm}$ diameter). Given the efficient diffusion of iodine as observed in Figs. 3 and 5, multiple wells for starch droplets were positioned at a larger distance of $7.5 \mathrm{~mm}$ from the KI chamber.

This device was filled with $1.5 \mathrm{~mL}$ of hexadecane, then $20 \mu \mathrm{L}$ of $10 \mathrm{mg} / \mathrm{mL}$ starch solution is pipetted in each of the five top wells, followed by $20 \mu \mathrm{L}$ of a $1 \mathrm{M}$ solution of $\mathrm{KI}$ in the middle chamber and finally $40 \mu \mathrm{L}$ of the brominegenerating reaction mixture (Table I) in the bottom chamber. As shown in Fig. 7A, when the two half-solutions were mixed in the bottom well, the droplet first turned yellow and then orange, indicating continuous formation of bromine. The bromine diffused through the hexadecane channel towards the KI chamber, which first coloured orange and then brown, indicating continuous triiodide ion generation as 
enabled by iodine formation through the halogen displacement reaction.

Iodine generation by diffusion-delivered bromine to the KI chamber was also evident from the purple colouring of the downstream channel network, shown in Fig. 7B. Upon arrival of the iodine in the top wells, the starch droplets slowly turned black because of the iodine association with the amylose molecules, thereby completing the bromine and iodine diffusion-facilitated reaction cascade. Colouring of the five top wells was not as rapid as in the devices described above because KI was omitted from the starch solution. Over the same time period, the colour of the bromine generation chamber reduced in intensity, indicating that this droplet had ceased to produce bromine due to depletion of one of the reaction components.

This experiment was repeated with the brominegenerating reaction mixture present in both the bottom and the middle chamber of the device. As seen in Fig. 7C, the bromine generated by both droplets could diffuse through the hexadecane channels towards the wells with the starch droplets, but this did not cause the starch to turn black. This control experiment thus confirmed that the final output (i.e. visualisation of the starch reaction) of this prototype reaction-diffusion network can only be realised with $\mathrm{KI}$ in the middle chamber. Without the halogen displacement reaction occurring in the middle chamber, the brominegenerating bottom chamber and the iodine-indicator top wells are not coupled, as there is no iodine produced.

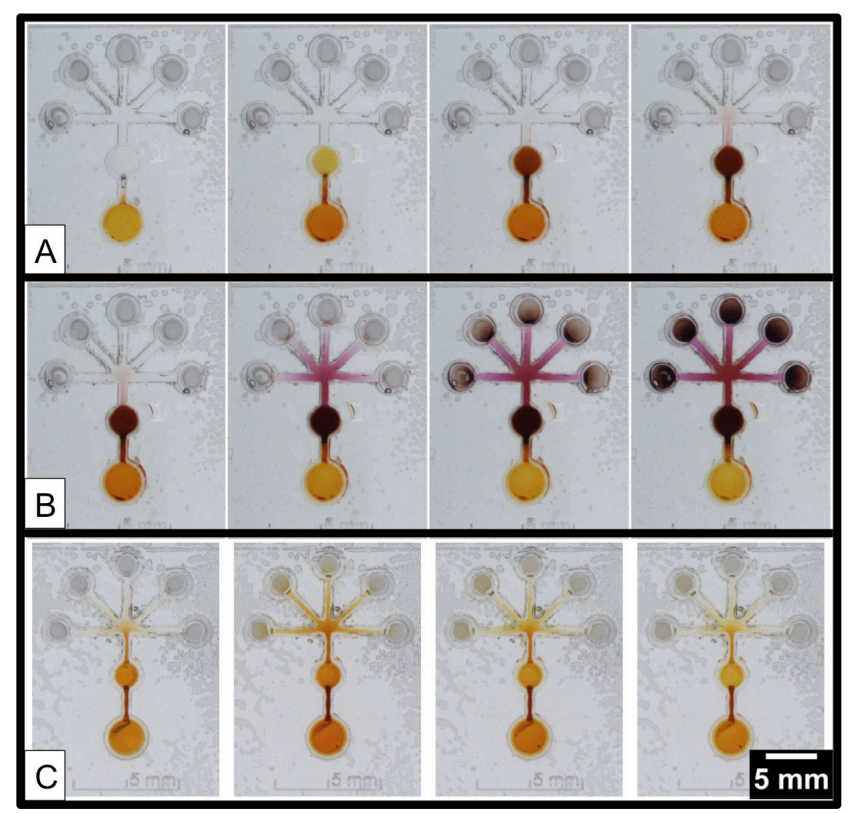

Fig. 7. Sequential reaction network with a bromine-generating source chamber at the bottom of the images, a centre chamber with KI solution where iodine can be formed through the halogen displacement reaction, and five top wells that contain starch for iodine detection. (A) Bromine diffusion from the source chamber triggers iodine formation and diffusion from the centre chamber (images at 0,10, 20, 30 mins). (B) Progression of iodine diffusion through the branched channel network causes colour development in the starch chambers due to the iodine-starch reaction (images at 30, 90, 150, 210 mins). (C) Control experiment with brominegenerating solution in both the bottom and the centre chamber shows bromine diffusion towards the starch chambers but no colouring of the starch chambers due to the absence of KI (images at 0, 30, 60, 90 mins).

\section{CONCLUSION}

The millifluidic prototypes described in this paper enabled the positioning of aqueous reaction mixtures at predefined locations that are physically connected by oil-filled channels. It was demonstrated that the reaction mixtures remain confined to the wells, which was achieved by designing the wells to be larger in diameter than the channels and by filling the wells to capacity, wall to wall, with aqueous solution. These networks were stable for many hours because a layer of oil covers the droplets, protecting the aqueous phase from evaporation even overnight. This long-term integrity of the two-phase system is an important precondition to study multi-layer reaction-diffusion networks.

Functional coupling between wells was achieved by halogen diffusion through the oil-filled channels. The optical transparency of the PMMA ensured ready visualisation of bromine and iodine synthesis and diffusion through the hexadecane, facilitating characterisation of their diffusion timescales and hence efficient optimisation of the reaction mixture compositions and of the geometries of the channels and wells. Potassium iodide as a halogen displacement reaction medium and starch solution as a well-known indicator dye for iodine enabled confirmation of bromine and iodine diffusion, and in the final three-component network, also presented a sequential chemical reaction network.

The design-to-experiment fabrication time of these prototypes is a couple of hours, while the consumables such as the PMMA itself are of minimal cost. This laser cutting fabrication method is thus well suited to investigate a wide range of network topologies, with channels and wells imparting structure on the reaction media. With a two-phase implementation, where wells contain the aqueous phase and channels contain the apolar phase, compositional and therefore functional compartmentalisation can be maintained.

In combination, the compartmentalisation and stability of the networks described here open up the path to a systematic experimental exploration of heterogeneous reaction-diffusion automata $[17,18]$. In the millifluidic size range supported by our fabrication technique, the diffusion speed of signalling molecules and ions will result in very slow progress of even elementary computations involving only simple combinations of sequencing, timing, and conditional triggers. Clearly such a mode of computation is not useful in the typical application context of conventional computing. Instead, we envisage application scenarios in simple control schemes for slow processes such as steering plant growth or the control of low-cost lab-on-chip devices [19] in which the time scale of operation is already diffusion limited. In many such cases a computation would need to run only once, and accordingly would not require replenishing the compartmentalised chemicals [20].

On a longer term perspective, we note that the early origins of computers can be traced to the sequencing, timing and conditional activation mechanisms of weaving looms, musical automata, and astronomic clocks [21] and that even present day microcontrollers dedicate significant hardware 
resources to these operations. Arguably, it is conceivable that progress in the implementation of reaction-diffusion computing will eventually lead to much more advanced "chemical controllers" composed of networks of chemically coupled droplets [22]. This direction of development will require a parallel reduction in size to reduce diffusion times. There is plenty of room for progress however: the sophisticated capabilities of biological tissues give an indication of what is physically and chemically possible.

\section{REFERENCES}

[1] A. M. Turing, "The chemical basis of morphogenesis," Phil. Trans. R. Soc. Lond. B, vol. 237, pp. 37-72, 1952.

[2] P. Ball, "Forging patterns and making waves from biology to geology: a commentary on Turing (1952) 'The chemical basis of morphogenesis'," Philos. Trans. R. Soc. B Biol. Sci., vol. 370, 20140218, 2015.

[3] L. Kuhnert, "Photochemische Manipulation von chemischen Wellen," Naturwissenschaften, vol. 73, pp. 96-97, 1986.

[4] J. Holley, A. Adamatzky, L. Bull, B. De Lacy Costello, and I. Jahan, "Computational modalities of Belousov-Zhabotinsky encapsulated vesicles," Nano Commun. Netw., vol. 2, pp. 50-61, 2011.

[5] P. H. King, J. C. Corsi, B.-H. Pan, H. Morgan, M. R.R. de Planque, and K.-P. Zauner, "Towards molecular computing: co-development of microfluidic devices and chemical reaction media," BioSystems, vol. 109, pp. 18-23, 2012.

[6] B. T. Ginn, B. Steinbock, M. Kahveci, and O. Steinbock, "Microfluidic systems for the Belousov-Zhabotinsky reaction," $J$. Phys. Chem. A, vol. 108, pp. 1325-1332, 2004.

[7] I. R. Epstein, "Coupled chemical oscillators and emergent system properties," Chem. Commun., vol. 50, pp. 10758-10767, 2014.

[8] M. Toiya, V. K. Vanag, and I. R. Epstein, "Diffusively coupled chemical oscillators in a microfluidic assembly," Angew. Chem. Int. Ed., vol. 47, pp. 7753-7755, 2008.

[9] H. Becker and C. Gärtner, "Polymer microfabrication technologies for microfluidic systems," Anal. Bioanal. Chem., vol. 390, pp. 89-111, 2008.

[10] D. Qin, Y. Xia, and G. M. Whitesides, "Soft lithography for microand nanoscale patterning," Nat. Protoc., vol. 5, pp. 491-502, 2010.

[11] J. N. Lee, C. Park, and G. M. Whitesides, "Solvent compatibility of poly(dimethylsiloxane)-based microfluidic devices," Anal. Chem., vol. 75, pp. 6544-6554, 2003.

[12] M. W. Toepke and D. J. Beebe, "PDMS absorption of small molecules and consequences in microfluidic applications," Lab Chip, vol. 6, pp. 1484-1486, 2006.

[13] P. H. King, C. H. Abraham, K.-P. Zauner, and M. R. R. de Planque, "Excitability modulation of oscillating media in 3D-printed structures," Artif. Life, vol. 21, pp. 225-233, 2015.

[14] M. Clugston and R. Flemming, Advanced Chemistry. Oxford: Oxford University Press, 2000, pp. 318-331.

[15] R. M. Noyes, R. J. Field, and E. Körös, "Oscillations in chemical systems. I. Detailed mechanism in a system showing temporal oscillations," J. Am. Chem. Soc., vol. 94, pp. 1394-1395, 1972.

[16] A. M. D. Wan, A. Sadri, and E. W. K. Young, "Liquid phase solvent bonding of plastic microfluidic devices assisted by retention grooves," Lab Chip, vol. 15, pp. 3785-3792, 2015.

[17] A. Adamatzky, B. De Lacy Costello, and T. Asai, Reaction-Diffusion Computers. New York: Elsevier Science, 2005.

[18] A. Adamatzky and B. De Lacy Costello, "Reaction-diffusion computing," in Handbook of Natural Computing, vol. III, G. Rozenberg, T. Bäck, and J. N. Kok, Eds. Berlin, Heidelberg: Springer, 2012, pp. 1897-1920.

[19] D. M. Cate, J. A. Adkins, J. Mettakoonpitak, and C. S. Henry, "Recent Developments in Paper-Based Microfluidic Devices," Anal. Chem., vol. 87, pp. 19-41, 2015.
[20] K.-P. Zauner and M. Conrad, "Parallel computing with DNA: Toward the anti-universal machine," in Parallel Problem Solving from Nature-PPSN IV, H.-M. Voigt, W. Ebeling, I. Rechenberg, and H.-P. Schwefel, Eds. Berlin, Heidelberg: Springer, 1996, pp. 696-705.

[21] T. Koetsier, "On the prehistory of programmable machines: musical automata, looms, calculators," Mech. Mach. Theory, vol. 36, pp. 589603, 2001

[22] G. Jones, P. H. King, H. Morgan, M. R. R. de Planque, and K.-P. Zauner, "Autonomous Droplet Architectures," Artif. Life, vol. 21, pp. 195-204, 2015. 